\title{
Artificial Intelligence Method for Electric Drives Mode Operating and Technical Condition Determination
}

\author{
Tatyana Kruglova ${ }^{1, *}$, Alexey Bulgakov ${ }^{2}$, Alexander Vlasov ${ }^{l}$, and Ivan Shmelev ${ }^{l}$ \\ ${ }^{1}$ South-Russian State Polytechnic University, 346428 Novocherkassk, Russia \\ ${ }^{2}$ Southwest State University, 505040 Kursk, Russia
}

\begin{abstract}
This paper proposes artificial intelligence method to determinate the status of electromechanical equipment by analysing the changes in the readings o parameters of its operating mode. As experimental results have revealed the dependence of wavelet transformation coefficients on the characteristic scales of functional and faulty motors under different loadsbased on which a neural classification network is developed to reveal the current state of the electromechanical equipment. Further studies have shown that any mother wavelet function can be used to implement the proposed method. The researches of the state of the drive under various loads confirm the correctness of the theoretical calculations and the adequacy of the model.
\end{abstract}

\section{Introduction}

Long-term operation of electrical equipment can arise various kinds of defects as a consequence of the wear and tear process. Operating the motors in poor technical condition of leads to financial losses related to unpredictable failure of equipment and a consequent violation of the technological process. One of the ways to solve the problem of sudden equipment failures is to monitor and evaluate its current status, identified by means of diagnostic methods, allowing to classify the current technical condition of the motor to one of the predetermined classes of diagnoses and to determine the locations of the defects that have arisen. The functional approach allows to identify the state of the drive during its operation. The selected diagnostic parameters should identify all the object faults that have occurred, should be simple to measure and analyse the outcomes. In contrast, these requirements are met by vibration, noise and current consumption. The traditional method of analysing such parameters is the Fourier transform [1], which has a number of significant drawbacks [2], not allowing its implementation for automatic diagnostics of electric drives operating under dynamic loads. As for main challenge stands the large amount of non-formalized qualitative information that cannot be used with conventional modelling methods. This issue can be addressed and solved using artificial intelligence methods.

\section{Materials and methods}

Long operation activity of the electrical motors at the large reversed loads can generate faults. One of the most simple and accessible methods of diagnosing the drive condition is the method of the spectral analysis of stator current signals allowing to carry out diagnostics of the electrical equipment and the connected mechanical devices. The received data are converted into the frequency domain using the Fourier transformation [1]. This transformation is widely used, however it has the following disadvantages:

1. The Fourier transform provides the frequency information, which is contained in the signal, however it cannot determine the time of occurrence of this frequency.

2. Limiting descriptiveness analysis of non-stationary signals: there is no possibility to analyze characteristics of the signal because the frequency-domain signal drop occurs over the entire frequency range of the spectrum. In addition the noise generated from "parasitic" highfrequency components render the readings very difficult to analyze.

3. Harmonic basis functions decomposition is not able to display signals with slope type rectangular pulses.

4. Simultaneously, producing temporal and frequency analysis is impossible.

5. Frequency analysis obtained using the Fourier transformation is complex and requires experienced specialist to understand it, which entails additional expenses.

These drawbacks do not make it possible to distinguish the faulty state of the electric drive from the change in its operating mode.To solve this problem, it is possible to apply wavelet analysis of signals, which considers the analysed time functions in terms of oscillations localized in time and frequency, providing a two-dimensional sweep of one-dimensional signals. In this case, the frequency and coordinate are considered as independent

\footnotetext{
* Corresponding author: tatyana.kruglova.02@mail.ru
} 
variable, which makes it possible to analyse the signals in two spaces at once. The wavelet functions of the basis allow us to concentrate the attention on certain local features of the analysed processes, which cannot be detected using traditional Fourier and Laplace transforms.

The wavelet transformation of a signal is represented in the form of a generalized series or the Fourier integral over a system of basis functions [3-5]

$$
\psi_{a b}(t)=1 / \sqrt{a} \cdot \psi\left(\frac{t-b}{a}\right),
$$

constructed from the mother (original) wavelet $\psi(t)$ possessing certain properties, due to the time shift operations $b$ and the time scale change $a$. The factor $1 / \sqrt{a}$ ensures that the norm of these functions is independent of the scaling number $a$. Small values, $a$ correspond to small scales $\psi_{a b}(t)$, or high frequencies $(\omega \sim 1 / \mathrm{a})$, large parameters $a$ - to large scale $\psi_{a b}(t)$, i.e. stretching the parent wavelet $\psi(t)$ и and compressing its spectrum. Thus, the wavelet scale, as a unit of the time-frequency representation of the signals, is inversely proportional to the frequency (Fig. 1).

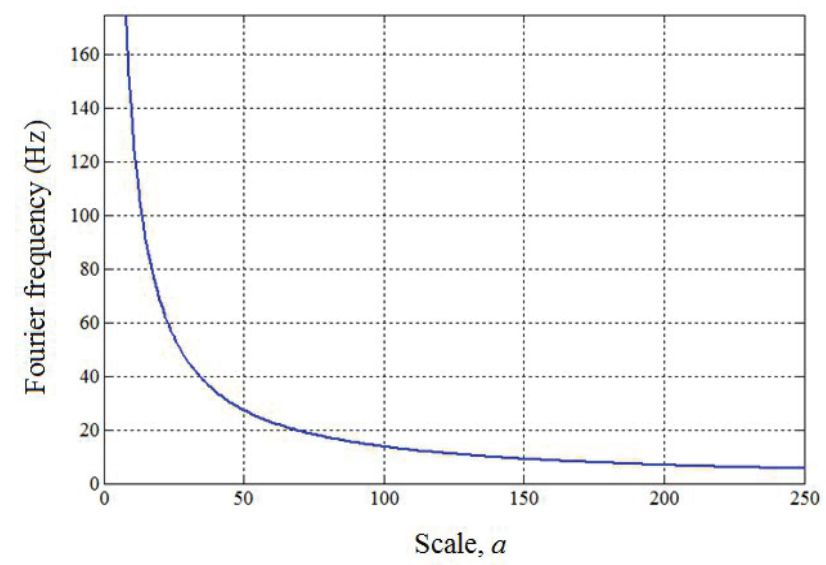

Fig. 1. Value for Fourier frequency with reference to the wavelet scale

The recalculation of the Fourier frequencies of the spectrum into the scale of the wavelet, according to the dependence in fig. 1, will allow signals analysis into wavelet space [6] and find new signal characteristics that cannot be detected using Fourier analysis.

\subsection{Experimental researches}

To analyse the state of the drive, the current signal, phase voltage and vibration speed of the new healthy motor, which is taken as the reference are required. Troubleshooting in the spectrum of the current signal is carried out at characteristic frequencies [6] by $\dot{t}$ comparing the current spectrum with the reference spectrum using artificial intelligence described later in the paper. For example, for a DC motor, the characteristic of Fourier transform frequencies are shown in Table 1.
Table 1. Characteristic frequencies of the current signal

\begin{tabular}{|c|l|}
\hline DC motor faults & \multicolumn{1}{|c|}{$\begin{array}{c}\text { Current (voltage) } \\
\text { signal frequency }\end{array}$} \\
\hline Commutation defects & $2 \cdot k \cdot p \cdot f_{r}$ \\
\hline Rotor defects & $2 \cdot p \cdot f_{r}, \quad k \cdot f_{r} \pm 2 \cdot p \cdot f_{r}$ \\
\hline Voltage ripple & $k \cdot f_{s}$ \\
\hline Stator defects & $k \cdot f_{r}$ \\
\hline
\end{tabular}

where $f_{s}$ - frequency of the network supplying the rectifier, $(\mathrm{Hz}) ; f_{r}-$ motor rotor speed, $(\mathrm{Hz})$; $k=1,2,3$ - number of current harmonic; $p$ - the number of poles.

The decomposition of the time current, voltage or vibration signals into a Fourier series and the analysis of the amplitudes at these characteristic frequencies reveals the observed object's faults. However, the analysis process is very laborious and will not distinguish the faulty state of the engine from a change in its operating mode. Therefore, for further analysis, it is necessary to recalculate the characteristic frequencies of the Fourier analysis into the scale of the wavelet. Any mother function can be chosen considering that of for a wavelet is achieved. Table 2 shows the result of the conversion to the scale of the Morlet wavelet for brushless DC motors PITTMAN 5413 at $3 \mathrm{~Hz}$ (Table 2).

Table 2. Ratio of the characteristic frequencies of the Fourier transform and the scale of the wavelet

\begin{tabular}{|c|c|c|}
\hline DC motor faults & $\begin{array}{c}\text { Fourier spectrum } \\
\text { frequencies }\end{array}$ & $\begin{array}{c}\text { Morlet wavelet } \\
\text { scale }\end{array}$ \\
\hline Commutation & 24 & 48 \\
defects & 48 & 24 \\
& 72 & 16 \\
\hline \multirow{3}{*}{ Rotor defects } & 24 & 48 \\
& 27 & 43 \\
& 30 & 39 \\
Voltage ripple & 33 & 35 \\
\hline & 50 & 23 \\
Stator defects & 100 & 12 \\
& 30 & 8 \\
\hline
\end{tabular}

A comparative analysis of the wavelet coefficient functions at characteristic frequencies for different operating modes reveals the general rule to diagnostic DC and AC motors using the aforementioned method (fig. 2). From the provided readings, it is visible that the wavelet coefficients of a serviceable unloaded engine have insignificant fluctuations at motor start-up and then the process is practically linearized. When a load appears, the oscillatory process at the start of the motor is clearer, but then decreases. However, it should be noted that the process is repeated with certain periodicity Although the process is stable, there is no significant increase in the amplitude of the oscillations with time. The coefficients of the wavelet transform of the faulty 
motor are much lower than those of the serviceable one and have constant oscillations-increasing with the load.
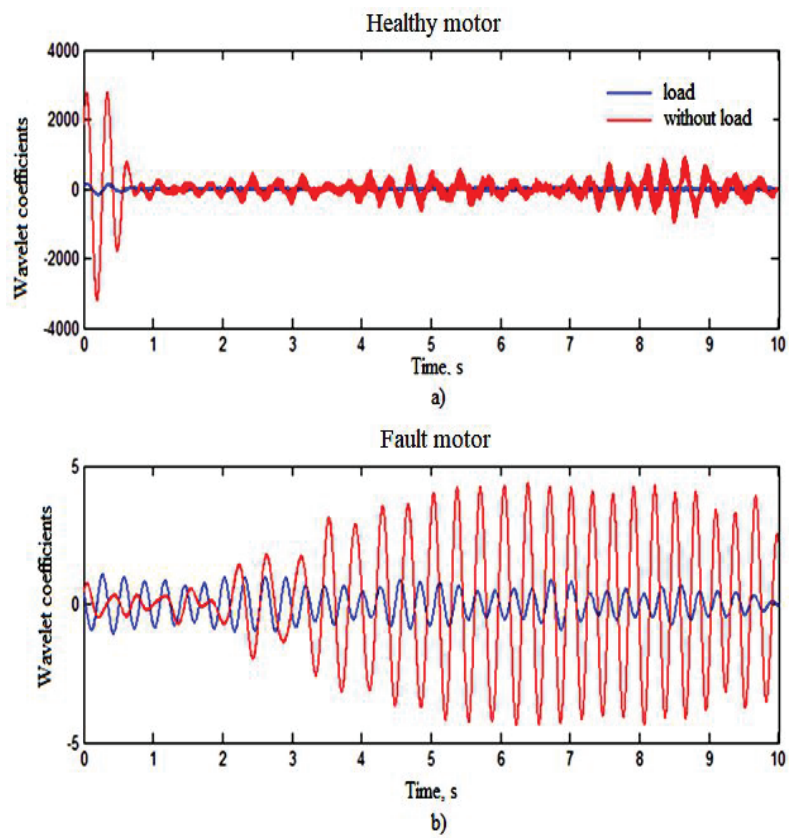

Fig. 2. Wavelet stator current signal: (a) healthy motor, (b) faulty motor

The carried-out analysis shows which wavelet values coefficients of current, voltage and vibration at the scales corresponding to malfunction has an identical characteristic appearance that can be used for electric drives mode operating and technical condition determination.

Fig. 3 illustrates the non-characteristic wavelet values. From this diagram it is visible, the signal has high density and small values wavelet coefficients. At the same time, the signal is regular and completely repeats with the given frequency. This type of signals is identical to all frequencies indicating failure in independence of technical condition of the motor

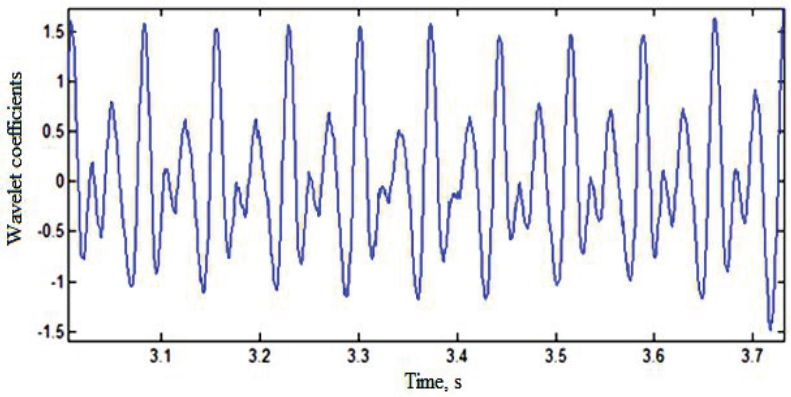

Fig. 3. The wavelet coefficients of the feeding tension of the serviceable and faulty engine at an non-characteristic scale

By analysing the results, we receive five characteristic signals for diagnosing. Signals of characteristic frequencies for the operational off-load and loaded engines (fig. 2), and also an unrepresentative signal (fig. 3), it is expedient to carry to a class "is serviceable" while faulty loaded and offload to a class "is faulty. This information can be used for simulation of a neural network to classify the technical condition of the engine.

\subsection{Identification of neural network for status classification}

For automatic detection of technical condition of the The purpose of the introduced neural network is to carry out automatic detection of technical condition of the electric drive without involvement of the specialist expert [7-19]. As basic data values, wavelet coefficients at a scale are used (fig. 2), characteristic of malfunction, and an uncharacteristic signal (fig. 3). As an entrance is set the matrix containing five lines of characteristic signals. The output of network is the diagnosis class: "1" - an object is serviceable, "2" - an object is faulty.

Fig. 4 illustrates the neural network used to classify the status of the drive

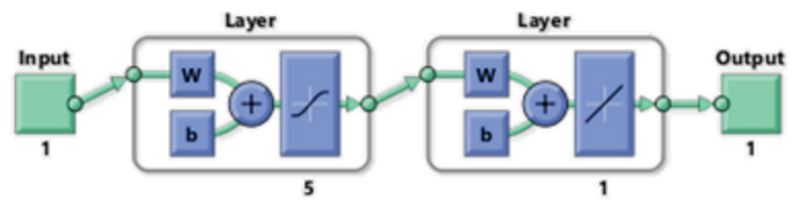

Fig. 4. Neural network for electric drive technical condition classification

The network consists of three layers: input, hidden and output. The hidden layer has five neurons with tangential function of activation, the output - one linear neuron.

For training of neural network is achieved using the Levenberg-Markvardt [11] algorithm intended for optimization of parameters of nonlinear regression models. The algorithm consists in consecutive approach of the set initial values of parameters to a required local optimum.

A training selection is a set of multiple couples of free variable of $x \in X^{M}$ (network inputs) and dependent variable of $y \in Y^{M}$ (a target vector). The functional dependence is the set representing a regression $y=f\left(\omega, x_{n}\right)$ continuously differentiable in the field of $W \times X$. The parameter $\omega$ is a vector of weight factors.

It is required to find such value of a vector $\omega$, which would deliver a local minimum of function of an error.

$$
E_{D}=\sum_{n=1}^{N}\left(y_{n}-f\left(\omega, x_{n}\right)^{2}\right.
$$

Before work of an algorithm is set the initial vector of weight coefficients $\omega$.

On each step of iteration, vector $\omega+\Delta \omega$ replaces the initial one. For an increment assessment of $\Delta \omega$ is used the linear approach of function:

$$
f(\omega+\Delta \omega, x) \approx f(\omega, x)+J \Delta \omega,
$$

where $J$ - Jacobean of function $f\left(\omega, x_{n}\right)$ in $\omega$. The matrix can be presented visually in the form: 


$$
J=\left[\begin{array}{ccc}
\frac{\partial f\left(\omega, f_{1}\right)}{\partial \omega_{1}} & \ldots \ldots & \frac{\partial f\left(\omega, f_{1}\right)}{\partial \omega_{R}} \\
\frac{\partial f\left(\omega, f_{N}\right)}{\partial \omega_{1}} & \cdots \cdots \cdots & \frac{\partial f\left(\omega, f_{N}\right)}{\partial \omega_{R}}
\end{array}\right]
$$

where $\omega=\left[\omega_{1} \ldots . \omega_{R}\right]^{T}$ is the vector of parameters.

The increment $\Delta \omega$ in a point $\omega$, the delivering minimum of $E_{D}$ is equal to zero. Therefore for finding ef the subsequent value of an incrementприращения $\Delta \omega$ we will equate to zero a vector of private derivative производных $E_{D}$ on weight $\omega$. For this purpose we will present (2) in the form

$$
E_{D}=|y-f(\omega+\Delta \omega)|^{2}
$$

where $y=\left[y_{1}, \ldots . . y_{N}\right]^{T}$ and

$$
f(\omega+\Delta \omega)=\left[f\left(\omega+\Delta \omega, x_{1}\right), \ldots . f\left(\omega+\Delta \omega, x_{N}\right)\right]^{T} .
$$

By transforming and differentiating equation (6):

$$
\begin{gathered}
\left.|y-f(\omega+\Delta \omega)|^{2}=(y-f(\omega+\Delta \omega))^{T} y-f(\omega+\Delta \omega)\right)=\text { (7) } \\
\left.=f^{T}(\omega+\Delta \omega) f(\omega)-2 y^{T} f(\omega+\Delta \omega)\right)+y^{T} y
\end{gathered}
$$

we can write the following:

$$
\frac{\partial E_{D}}{\partial \omega}=\left(J^{T} J\right) \Delta \omega-J^{T}(y-f(\omega))=0
$$

Hence, to find value $\Delta \omega$ it is necessary to solve system of the linear equations

$$
\Delta \omega=\left(J^{T} J\right)^{-1} J^{T}(y-f(\omega)) .
$$

As the number of conditionality of a matrix of $J^{T} J$ is a square of number of conditionality of a matrix of $J$, a matrix $J^{T} J$ can be degenerate.

$$
\Delta \omega=\left(J^{T} I\right)^{-1} J^{T}(y-f(\omega)),
$$

where $I$ - single matrix.

This parameter is appointed on each iteration of an algorithm. If the value of an error $E_{D}$ decreases quickly, $\lambda$ reduces this algorithm to the Gauss-newton algorithm. The algorithm stops in case the increment $\Delta \omega$ in the subsequent iteration is less than present value or if the vector of weight coefficients delivers an error $E_{D}$, smaller the set size or if the number of cycles of training of neural network is exhausted.
The value of a vector $\omega$ on the last iteration is considered required.

The learning disabilities of the neural network are presented in fig. 5
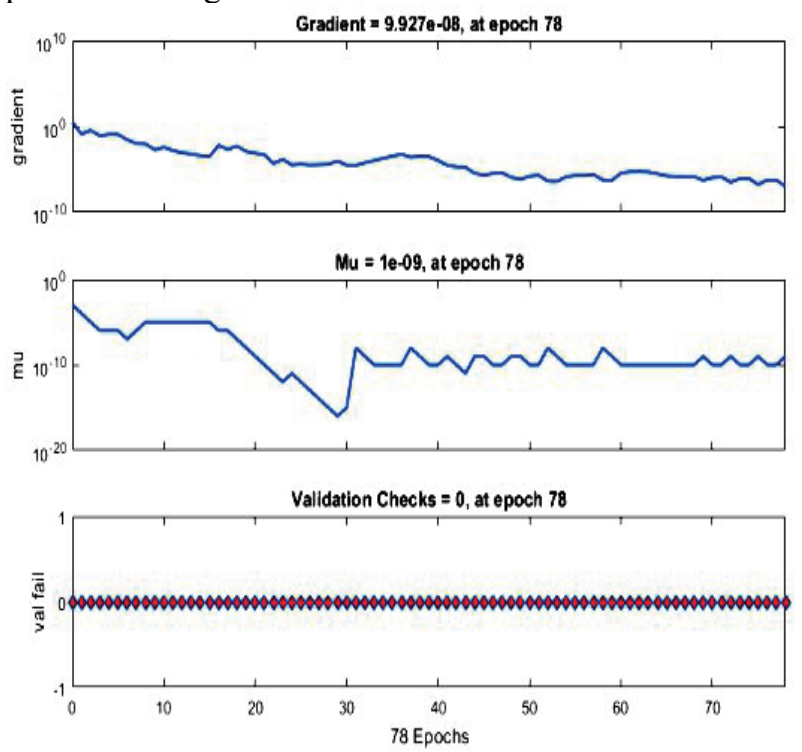

Fig. 5. Neural network training result for technical condition classification

The unloaded and loaded condition analysis of the drive (DC motors PITTMAN 5413, rotating speed of the motor of resistance of $1 \mathrm{~Hz}$ ) showed that 386, 193 and 129 scales of wavelet expansions belong to the class "2" and all remaining scales to the class "1", which demonstrates the existence of failure of the stator.

Similar tests were executed on the serviceable and faulty motor (Frequency of $0,2 \mathrm{~Hz}, 0,4 \mathrm{~Hz}, 0,6 \mathrm{~Hz} 0.8$ Hz.) A similar experiment was made for Daubechies wavelet of 10-th order, Haar, the Mexican hat and Gauss. Recalculation of frequencies of Fourier of conversion to scales of these wavelet is executed. Substitution wavelet coefficients on data of characteristic scales in the trained neural network allowed to identify failure of an object unmistakably. The results certify to correctness of theoretical calculations and adequacy to the developed neural network model of diagnosing.

The provided neural network algorithm ensure the distinguishing between functional and faulty engine irrespective of the load.

To determine the technical condition and operating mode of the motors, an advanced neural network of the following structure is introduced (fig. 6).

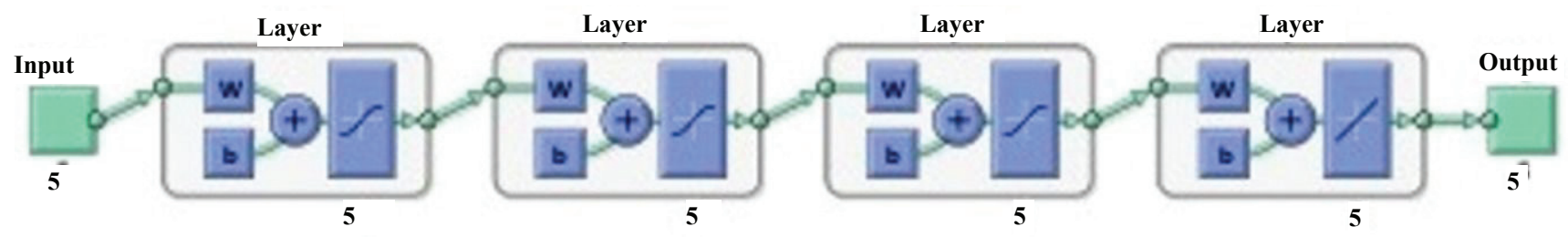

Fig. 6. Neural network for electric drive technical condition and mode operation classification 
The neural network s consists of five inputs, four hidden layers of with five neurons in each and five outputs. The goal of the neural network training is to distribute the five input signals (fig. 2, 3) to four classes: «11» - functional unload, «12» - functional loaded, «21» - faulty unload, «22» - faulty loaded. For neural network training the Levenberg-Markvardt [201] algorithm was used. The learning results of the neural network are presented in table 3 and fig. 7 respectively.

Table 3. Results of neural network for technical condition and operating mode classification

\begin{tabular}{|l|c|}
\hline \multicolumn{1}{|c|}{ Signals } & Network Results \\
\hline Functional unload, (fig. 2,a) & 11 \\
\hline Functional loaded, (fig. 2,a) & 12 \\
\hline Uncharacteristic signal (fig. 3) & 11 \\
\hline Faulty unload, (fig. 2,b) & 21 \\
\hline Faulty loaded, (fig. 2,b) & 22 \\
\hline
\end{tabular}
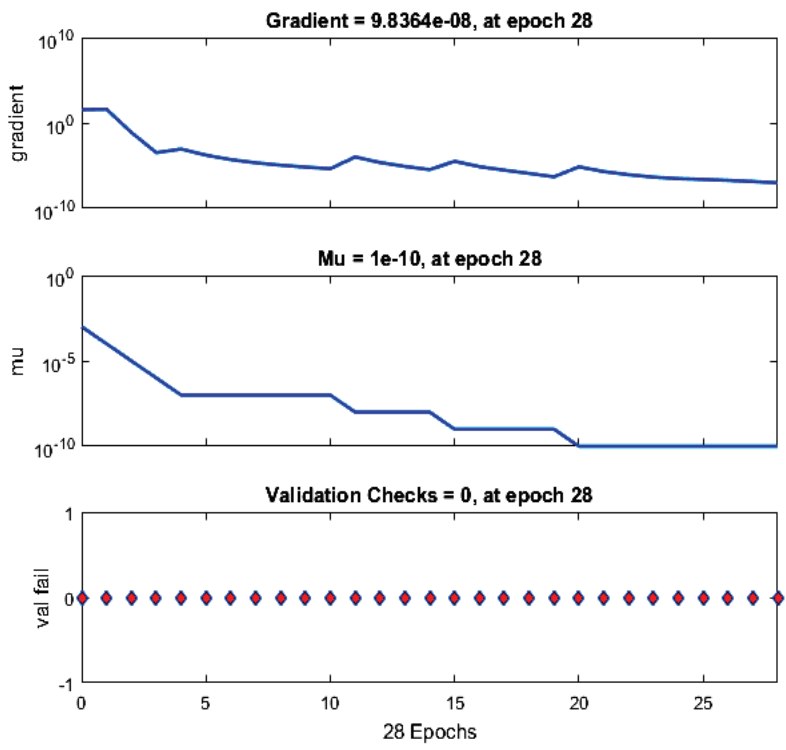

Fig. 7. Neural network training result for technical condition and operation mode determination

\section{Conclusions}

The artificial intelligence method of diagnosing of the electric drive allows with a high precision to find malfunctions of an observed electromechanical object. The realization of this method is based on wavelet transformation and neural networks. The validity of the theoretical calculations and adequacy of model are endorsed with simulation results based on specific electromechanical system.

\section{References}

1 Körner T.W. Fourier Analysis, CUP (1988)

2 Daubechies I. The wavelet transform time-frequency localization and signal analysis, IEEE TIT 961-1004 (1992)
3 Kruglova T.N. Intelligent Diagnosis of the Electrical Equipment Technical Condition, PE, 129, 219-224 (2015)

4 Peter W. Tsea, Wen-xian Yangb, Tama H.Y., JSV 277, 1005-1024 (2004)

5 Liu B., Ling S.F., MSSP, 13 (1), 145-162 (1999)

6 Nandi S., Hamid A. T., and. Li X., IEEE Conf J \& M., 20 (4), 719-729 (2005)

7 Yoon-Seok Lee, Kyung-Tae Kim, JinHur., IEEE TM, 50(2), 7022004-7022004 (2015)

8 Shakouhi M. Mohamadian M. Afjei E., IEEE TIE, 62 (3), 1400-1409 (2015)

9 Penga Z.K, Peter W. T., Chub F.L., MSSP, 19, 974988 (2005)

10 Awadallah M.A. Morcos M.M., IEEE TECC, 20(1), 2460-247 (2005)

11 Shen Z., Chen X., Zhang X., He Z., Measurement, 45 (1), 30-40 (2012)

12 Kliman G.B. Stein J., EMPS, 20 (5), 463-474 (1992)

13 Jiang L., Liu Y., Li X., Tang S., Measurement, 44 (7), 1284-1292 (2011)

14 Betta G., Liguori C., Pietrosanto A., Measurement, 30 (2), 129-138 (2001)

15 Dister C.J., Schiferl R., Proceedings of the IEEE IAS AMC, 1, 312-318 (1994)

16 Toliyat H.A., Lipo T.A., IEEE TEC, 10, 241-247 (1985)

17 Toliyat H.A., Lipo T.A., IEEE TEC, 10, 241-247 (1985)

18 Siddique A, Yadava GS, Singh B., EC IEEE Trans, 20(1), 106-114 (2005)

19 Medoued A, Lebaroud A, Boukadoum A, Boukra T, Clerc G. (SDEMPED), 2011 IEEE Int. symp. IEEE, 525-528 (2011)

20 Andersen T.J. Wilamowski B.M., WCNN, 1 687-690 (1995)

\footnotetext{
* Corresponding author: tatyana.kruglova.02@mail.ru
} 